\title{
Modeling of viscous friction on structures in beams with spherical plain bearings in the plan links
}

\author{
Djekine $^{1}$, Guy Edgar Ntamack ${ }^{1}$, Claude Armand Moubeke ${ }^{1, ~ *, ~ S y l v a i n ~ E r i c ~ K e l m a m o ~ S a l l a b o u i ~}{ }^{1}$, \\ Sâad Charif D'Ouazzane ${ }^{2}$
}

${ }^{1}$ Groupe de Mécanique et des Matériaux, GMM, Département de Physique, Faculté des Sciences, Université de Ngaounderé, B.P. 454

Ngaoundéré - Cameroun

${ }^{2}$ Laboratoire de Mécanique, Thermique et Matériaux, LMTM, Ecole Nationale de l'Industrie Minérale, ENIM, B.P. 753 Rabat, Maroc

\section{Email address:}

claudemoubeke@yahoo.fr (C. A. Moubeke)

\section{To cite this article:}

Djekine, Guy Edgar Ntamack, Claude Armand Moubeke, Sylvain Eric Kelmamo Sallaboui, Sâad Charif D’Ouazzane. Modeling of Viscous Friction on Structures in Beams with Spherical Plain Bearings in the Plan Links. American Journal of Mechanics and Applications. Vol. 2, No. 2, 2014, pp. 10-13. doi: 10.11648/j.ajma.20140202.12

\begin{abstract}
In the calculation of structures in beams with spherical plain bearings (SBSPB) static bindings, movements are continuous, while rotations are staple to a node where a ball. is located in Dynamics, in several calculation codes, and mechanical frictions are often supposed to be neglected. In this paper, we proposed a modeling of the SBSPB which takes into account the friction during the SBSPB vibrations and also in the calculation of the degrees of freedom at the points of these structures. Hence, mathematics equations were established and their resolution by the modal method was proposed. Static analysis of the results showed that there is continuity of translations, and discontinuity of rotations in spherical nodes. In two dimensional dynamic approaches, the results obtained, are represented in the form of curves and their analysis emphasizes the influence of links bearings and viscous frictions during the vibration of the structure.
\end{abstract}

Keywords: Ball, Modeling, Analytical, Static, Dynamic, Structures Beams Planes

\section{Introduction}

The ball is a spherical piece of steel used as a link in the organs of machine someone wants to steer in all direction [1].Spherical bearings are thus used as joints in robotics. In civil engineering; the bearings allow relaxation and stabilization of movements in bridges [1]. The problem in the modeling of the SBSPB is the determination of the rotation values in nodes where they are placed. In this contribution we will evaluate two-dimensional static and dynamic different degrees of freedom in the SBSPB plan, taking into account the viscous friction. In this paper, the first part is related to modeling of bearings in the SBSPB and the static condensation method that allows determination of generalized displacement. The second part is devoted to the study of vibration of mechanical systems of beams structures in two dimensions. We booked the last part to the analysis and discussion of our results.

\section{Theoretical Considerations}

\subsection{Modeling of Structures in Beams in Two Dimensions with Ball Links}

In beams structure, each component can be adapted to by a two-node linear element, as shown in the following figure [2]:

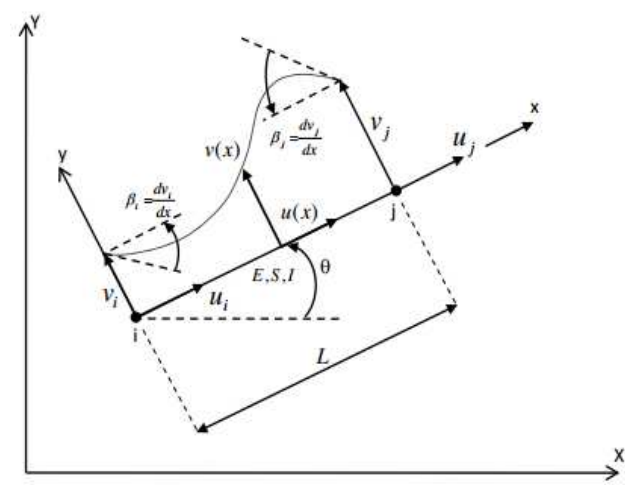

Figure 1. Beam Elements at 2 knots in the plan (xoy). 
In the plan, we can work with elements of two nodes $(i, j)$ and in each node; we consider three levels of freedom $\left(u_{i}, v_{i}, \beta_{i}, u_{j}, v_{j}, \beta_{j}\right)$ where:

$u$ : moving along the axis (ox);

$v$ : moving the y-axis (oy).

$\beta$ : rotation around the z-axis (oz).

$\theta$ : angle to determine the position of the element in the space.

Each beam element is characterized by the following geometric constants:

- L: length;

- S: section;

- E: modulus of longitudinal elasticity;

- $\mathrm{I}_{\mathrm{Oz}}$ moment of inertia along the axis (oz).

In the finite element method, calculation of widespread displacement, through the evaluation of stiffness matrices and the elemental strength matrices; and then, the Assembling of these matrices and vectors [2, 3]. In addition, problems in two dimensions, should take into consideration the spatial position of each element, therefore, must assess the transition matrices of the local coordinate system to the global coordinate system, which involves the position of various components in space [3].

\subsection{Static Rotations Calculations}

To calculate the displacements and rotations, we used the techniques outlined in references [4-6]. The specificity of these methods is the calculation of the rotations at the level of spherical nodes. At these nodes, there are two rotations and we calculate them in two different ways. The calculation of spherical nodes was done locally by static condensation technique [7] which consists of disrupting the elemental stiffness matrix based on the position of the ball node. Subsequently it was with the disrupted matrix that Assembling of matrices was done to obtain the global stiffness matrix. Calculations of the built-in nodes and other widespread movements were done with stiffness matrices and global strength vectors. Through illustration, we considered the structure of figure 2 without linking ball and those of figures 3, 4, 5 and 6 with a binding node 3 ball. The calculation of displacement and rotation at node 3 of the structure on figure 2 was done after assembling the elemental stiffness matrices without any disturbance. Regarding the structure on figure 3 , it was modeled in the following three ways: by putting the ball on the element (13 ), by putting the spin on the element (2-3), or by putting the ball on the element $(3-4)$. According to calculations developed in references [5,6], global rotations of node 3 for figures 4,5 and 6 are identical, if the framework is discredited by the same elements.

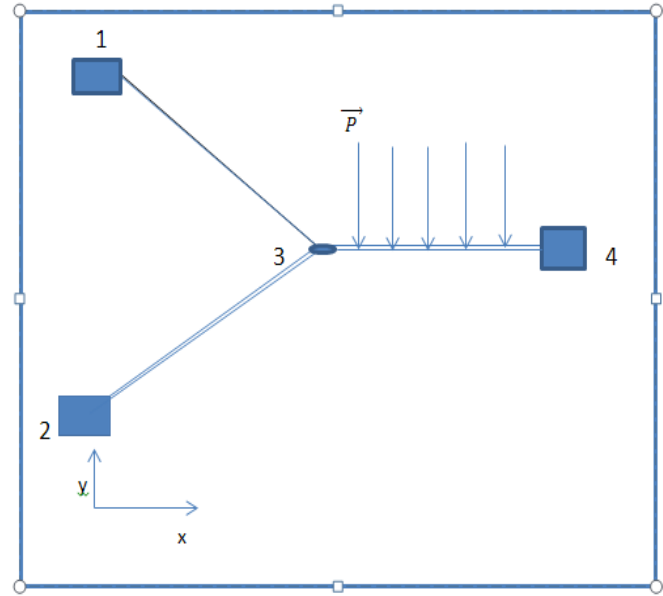

Figure 2. Structure in beams without binding node3ball.

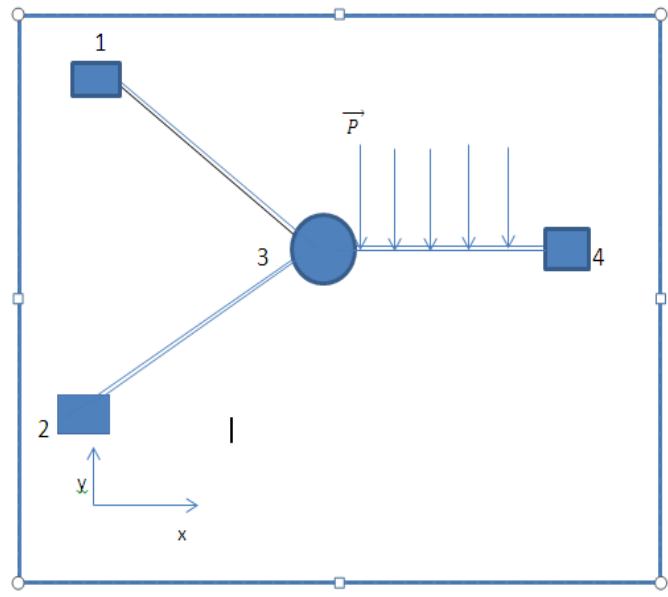

Figure 3. Structure in beams with binding node 3 ball.

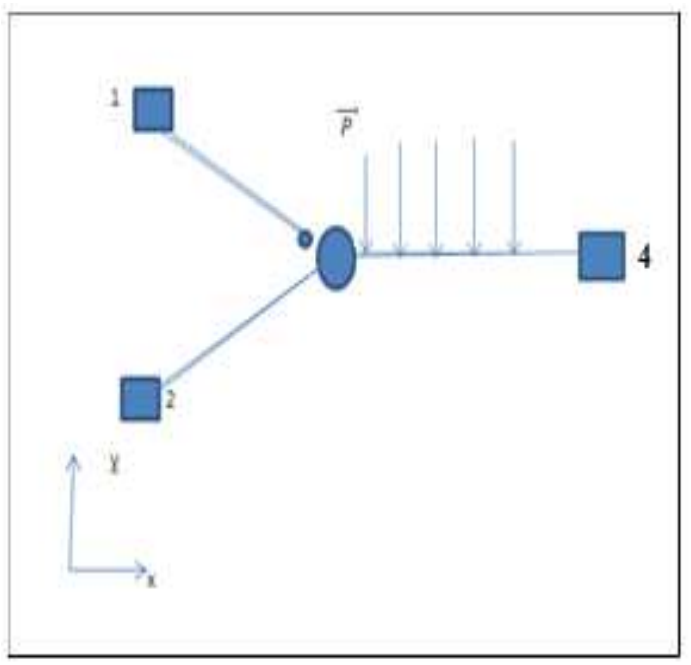

Figure 4. Spherical element (1-3). 


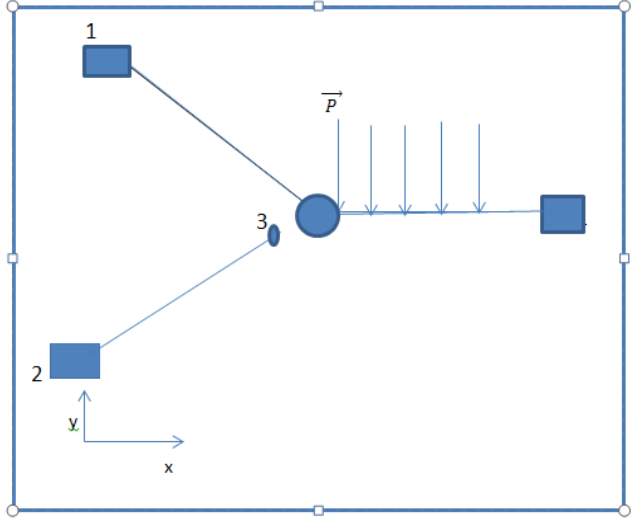

Figure 5. Ball on the element (2-3).

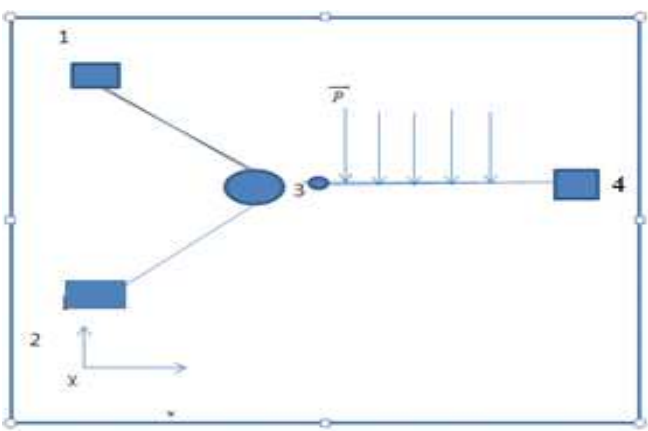

Figure 6. Spherical element (3-4).

For each of these models, two rotations related to node (3) were calculated the rotation of the ball node and the rotation of the built-in node [5].

\subsection{Calculation of Dynamic Rotations}

In Dynamics, the equations of motion of beams structures can be expressed in the following form:

$$
M \ddot{U}+C \dot{U}+K U=F(t)
$$

Where:

- $\mathrm{M}$ is the matrix mass $\mathrm{M}$;

- C the matrix (viscous) damping;

- $\mathrm{K}$ is the stiffness matrix;

- $\mathrm{F}(\mathrm{t})$ is the external strength vector;

- $U$ is comprised of the set of degrees of freedom.

This system of equation resolution technique has been developed in references $[5,6]$. It takes into account the friction method. The difficulty in modifying these systems is the decoupling of equations. There are several techniques to decouple these equations. In this contribution, we used the modal method [3]. The particularity of the equations obtained in this work is the integration of the bearings in the structures. This is justified by the disturbance matrices masses and the front damping matrices well as the diagonalization to decouple them [8]. Then we applied the classic modal method to solve the final equations obtained.

\section{Results and Discussions}

In the simulations, a beam IPN feature iron was chosen. $\mathrm{E}=210000 \mathrm{MPa}, \rho=7850 \mathrm{~kg} / \mathrm{m}^{3}, \mathrm{I}_{\mathrm{Oz}}=77.67 \mathrm{~cm}^{4}, \mathrm{~S}=7.57 \mathrm{~cm}^{2}$, $\mathrm{L}=10 \mathrm{~m}$. In addition, components were identical in all the structures.

\subsection{Static Resolution}

To present the effects of linkages bearings, we had simulations with structures as represented in figures 4,5 and 6 . The results obtained are summarizing in table 1 for each structure:

Table 1. Shift values generalized to node 3 static.

\begin{tabular}{lcccc}
\hline & Fig. 2 & Fig. 3 & Fig. 4 & Fig. 5 \\
\hline$u_{3}(\mathrm{~m}) / 10^{-3}$ & & & & \\
$\mathbf{v}_{3}(\mathrm{~m}) / 10^{-3}$ & -1.039 & -1.020 & -1.019 & -1.019 \\
$\boldsymbol{\theta}_{3}(\mathrm{rad}) / 10^{-3}$ & -5.710 & & & \\
$\boldsymbol{\theta}_{3}(\mathrm{rad}) / 10^{-3}$ & & -0.050 & -0.0507 & -0.0503 \\
\hline
\end{tabular}

According to data in table 1 , the rotation of the rigid node was more important than that of the spherical nodes. On elements (1-3), (2-3) or the component (3-4), we virtually had the same values of rotations. Therefore, we deduced the joints soften structures. According to our modeling; the position of patella on an element was irrelevant on the behaviour of the structure. Furthermore, the rotations were discontinuous, though the transverse moving at nodes structures were continuous.

\subsection{Dynamics Resolution}

We considered once more structures in figures 2 and 3 . The conditions of simulations are as follows:

$u_{0}=v_{0}=0.5 \mathrm{~m} ; \dot{u}_{0}=\dot{v}_{0}=1 \mathrm{~m} / \mathrm{s}, \quad \theta_{0}=0.5 \mathrm{rad} ; \dot{\theta}_{0}=0.5 \mathrm{rad} / \mathrm{s}$, under harmonic vibration amplitude load $P_{0}=10 \mathrm{~N}$ and in the case of small oscillations with shock absorber factor $\varepsilon=0.005$. We got the curves illustrated in figures 7 :

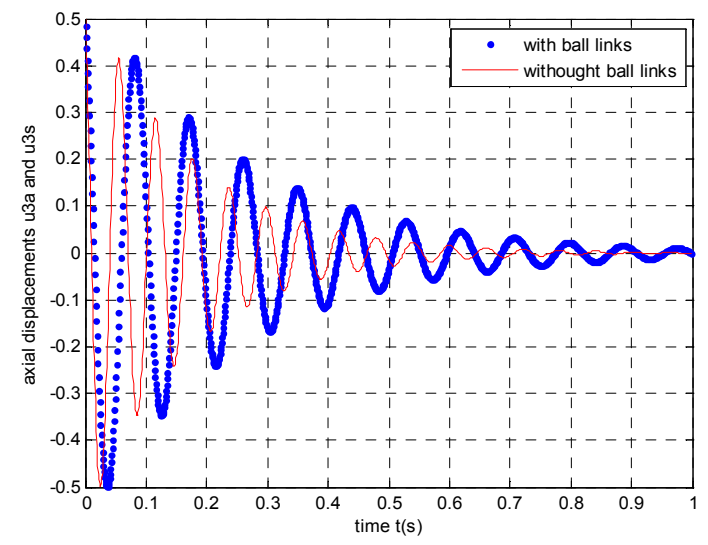

Figure 7. Curves of axial displacement $u_{3 a}(t)$ and $u_{3 s}(t)$ to node 3, free structure with friction. 


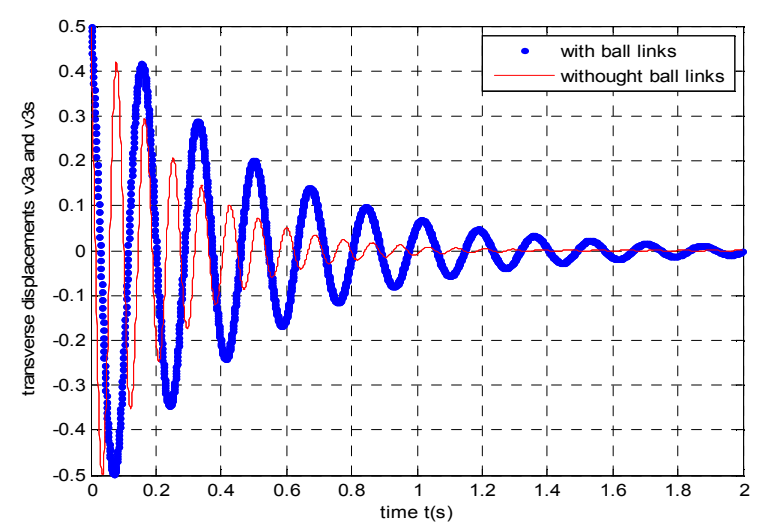

Figure 8. The transverse displacement curves $v_{3 a}(t)$ and $v_{3 s}(t)$ to node 3 , free structure with friction.

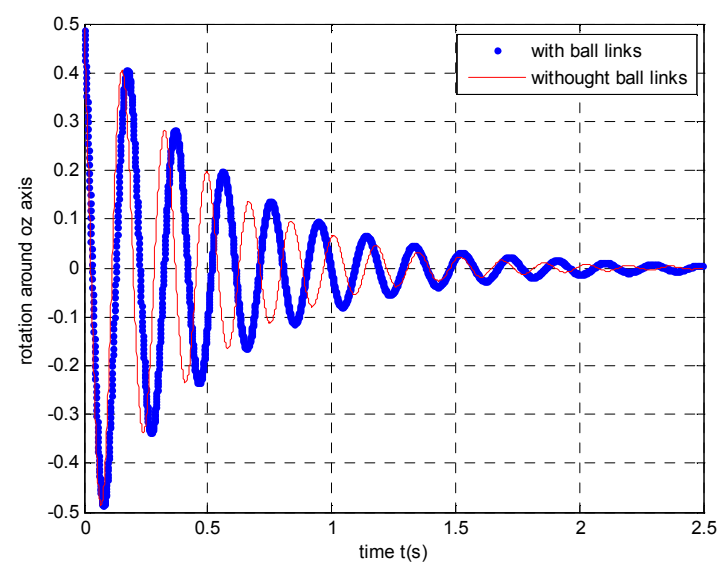

Figure 9. Curves of rotations $\theta_{3 a}(t)$ and $\theta_{3 s}(t)$ node 3 , free structure with friction.

For failure in service on figures 7 to 9 , the amplitude of the degrees of freedom of structures was always higher when they involved spherical plain bearings. More periods of structures vibration with links bearings were higher than those without these links. In figure 10, the amplitude and period of the structure without binding was more important than the structure without links. Hence, the rupture risk in the structures without bonds was higher at the beginning of the movement. As the result, that links joined soften movements and posed less risk.

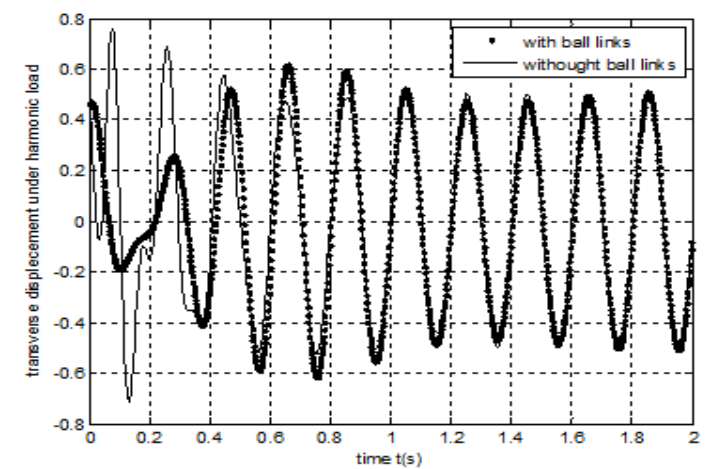

Figure 10. Curves of transverse displacements $v_{3 a}(t)$ and $v_{3 s}(t)$ at node 3 , amortized structure under harmonic loads.
At the end, in case of vibration damped under actual harmonic loads (fig. 10), we observed that transverse displacements were first amortized in the transition zone, and then, sinusoidal later in the stationary area.

\section{Conclusion}

This contribution was to propose a modeling of the SPLR method to study their static and dynamic behaviour. With our model, we found that static is in good continuity of movement axes (Ox) and (Oy), and rotation in recessed nodes. On the other hand, for nodes with links bearings, we had the continuity of the movement axes (Ox) and (Oy), and the discontinuity in rotation. Moreover, dynamic consideration permitted to prove the bearings soften leather SBSPB structures and extended their breaking period.

\section{References}

[1] P.H. Trompette, «Mécanique des structures par méthode des éléments finis, statique et Dynamique». Masson, Paris Milan Barcelone Bonn, 1992.

[2] J.F. Imbert, «Analyse des structures par éléments finis», 3ème édition de l'école nationale supérieure de l'aéronautique et de l'espace, 111, rue Nicolas-Vauquelin 31100 Toulouse, 1991.

[3] H. Bouabid, S. Charif d'Ouazzane, O. Fassi-Fehri, K. Zinedine A, «Representation of swivel joints in computing tridimensional structures», 3ème Congrès de mécanique, Tétouan, Apr. 1997.

[4] G.E. Ntamack, M. Dawoua Kaoutoing, T. Beda, S. Charif D'Ouazzane. "Modeling of swivel joint in two dimensional beams frameworks". IJST, 3, 1, 2013, p. 21-25.

[5] M. Dawoua Kaoutoing, G.E. Ntamack, K. Mansouri, T. Beda, S. Charif D'Ouazzane "Modeling of the damped oscillations of the viscous beams structures with swivel joints for harmonic mode". IJRET, vol. 02, Issue 07, 2013, p. 151-154.

[6] R. J Guyan, «Reduction of stiffness and mass matrix», AIAA, vol. 3 p. $380,1965$.

[7] H. Oudin, «Vibrations en petites perturbations des systèmes mécaniques», 2012. 